\title{
A Nutrigenetic approach to examine the relationship between vitamin $B 12$ status and cardio-metabolic traits in multiple ethnic groups - findings from the GeNulne Collaboration
}

\section{Article}

Published Version

Creative Commons: Attribution 4.0 (CC-BY)

Open Access

Vimaleswaran, K. S. and Surendran, S. (2021) A Nutrigenetic approach to examine the relationship between vitamin B12 status and cardio-metabolic traits in multiple ethnic groups findings from the GeNulne Collaboration. Nutrition Bulletin. ISSN 1471-9827 doi: https://doi.org/10.1111/nbu.12494 Available at https://centaur.reading.ac.uk/97035/

It is advisable to refer to the publisher's version if you intend to cite from the work. See Guidance on citing.

To link to this article DOI: http://dx.doi.org/10.1111/nbu.12494

Publisher: Wiley-Blackwell

All outputs in CentAUR are protected by Intellectual Property Rights law, including copyright law. Copyright and IPR is retained by the creators or other copyright holders. Terms and conditions for use of this material are defined in the End User Agreement. 


\section{www.reading.ac.uk/centaur}

\section{CentAUR}

Central Archive at the University of Reading

Reading's research outputs online 


\title{
A nutrigenetic approach to examine the relationship between vitamin $B \mid 2$ status and cardio-metabolic traits in multiple ethnic groups: Findings from the GeNulne Collaboration
}

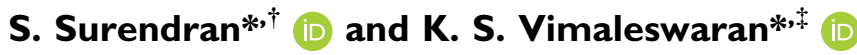 \\ *Hugh Sinclair Unit of Human Nutrition, Department of Food and Nutritional Sciences, University of Reading, Reading, UK; \\ †Faculty of Health and Medical Sciences, School of Biosciences and Medicine, University of Surrey, Guildford, UK; \\ *Institute for Food, Nutrition and Health (IFNH), University of Reading, Reading, UK
}

\section{Abstract}

Correspondence: Prof. Karani Santhanakrishnan Vimaleswaran, Professor of Nutrigenetics \& Nutrigenomics, Hugh Sinclair Unit of Human Nutrition, University of Reading, Reading, RG6 6DZ, UK. E-mail: v.karani@reading.ac.uk

Dr. Shelini Surendran, Teaching Fellow, Biosciences Foundation, School of Biosciences and Medicine, University of Surrey, Guildford, Surrey, GU2 7XH, UK.

E-mail: s.surendran@surrey.ac.uk

\begin{abstract}
Low vitamin B12 concentration has been shown to be a risk factor for cardiometabolic traits in numerous observational studies; however, the relationship has remained inconsistent. It is possible that certain genotypes jointly contribute to cardio-metabolic diseases and vitamin B12 deficiency, and these may be modulated by dietary factors. The main objective of this article is to summarise the findings from the GeNuIne (Gene-Nutrient Interactions) Collaboration on the effect of gene-nutrient interactions on vitamin B12 concentrations and cardio-metabolic disease risk factors in population-based studies from different ethnic groups. Interactions between vitamin B12-related single-nucleotide polymorphisms (SNPs) and protein energy intake $(\%)$ on waist circumference $\left(P_{\text {interation }}=0.002\right)$ and body fat percentage $\left(P_{\text {interaction }}=0.034\right)$ were observed in Sri Lankan and Indonesian populations, respectively. In the study in Brazilian adolescents, the metabolic and vitamin B12-related SNPs showed a significant interaction with dietary carbohydrate and protein intakes on oxidised low-density lipoprotein cholesterol and homocysteine concentrations, respectively. In the Asian Indian population, an association between obesity-related SNPs and vitamin B12 concentrations $(P=0.018)$ was observed. In summary, these studies in multiple ethnic groups show that the association between genetically low vitamin B12 concentrations and cardio-metabolic traits may be modified by dietary intake. Further studies utilising larger sample sizes are needed to confirm or refute our findings.
\end{abstract}

Keywords: GRS, metabolic traits, nutrigenetics, obesity, SNP, vitamin B12 pathway 
deficiency (Green et al. 2017). Vitamin B12 deficiency can also be caused by having inadequate amounts of intrinsic factor (IF), gastric atrophy, intestinal disease, gastric surgery, bacterial overgrowth in the small intestine, alcohol consumption, a tapeworm infection, drug-nutrient interactions and some genetic defects (Institute of Medicine 2000; Allen 2008; O’Leary \& Samman 2010). Additionally, alarmingly high prevalence rates of low plasma vitamin B12 status have been recognised to exist in certain regions of the world including the Indian subcontinent, Mexico, Central and South America and selected areas in Africa (Stabler \& Allen 2004).

Vitamin B12 functions as a coenzyme of several key enzymes in one-carbon metabolism, which in turn are essential for nucleotide synthesis and methylation reactions (Selhub 2002). The two active forms of vitamin B12, methylcobalamin and 5-deoxyadenosylcobalamin, are essential coenzymes for the homeostasis of methionine and methylmalonic acid (MMA), respectively (Koury \& Ponka 2004; Sobczyńska-Malefora et al. 2014). Observational studies have shown that low vitamin B12 concentrations are accompanied by a wide range of chronic diseases and conditions, including obesity, insulin dysregulation and adverse cardiometabolic outcomes (Baltaci et al. 2013; Mahalle et al. 2013; Adaikalakoteswari et al. 2014; Narang et al. 2016; Chakraborty et al. 2018; Guarnizo-Poma et al. 2018; Jayashri et al. 2018). The aetiology between low vitamin B12 concentrations and metabolic phenotypes is still under debate and could be the result of several mechanisms. Vitamin B12 deficiency could possibly lead to adipocyte dysfunction by modulating lipid metabolism and enhancing cellular inflammation (Kumar et al. 2013). Several studies have suggested that vitamin B12 deficiency is more prevalent in individuals who are obese as result of either low dietary vitamin B12 consumption (Thomas-Valdés et al. 2017; Sun et al. 2019) or increased vitamin B12 demands secondary to an increased surface area of the body (Wall 1998; Pinhas-Hamiel et al. 2006).

Genetic studies have implicated several gene loci associated with circulating vitamin B12 (Surendran et al. 2018). To date, only three Mendelian randomisation studies have explored the relationship between a genetically determined decrease in serum vitamin B12 concentrations on cardio-metabolic traits such as body mass index (BMI) (Allin et al. 2017), blood pressure (Husemoen et al. 2016) and cardio-metabolic risk (Moen et al. 2018). However, these studies were unable to support the causal role of decreased serum vitamin B12 concentrations with these cardio-metabolic traits. Further to this, dietary factors play an important role in the development of vitamin B12 deficiency, but this relationship may differ across countries, due to the variation in food consumed worldwide (Vimaleswaran 2017). Studies have shown that the intrauterine imbalance of vitamin B12 and folate can affect DNA methylation and 'programme' the offspring to develop metabolic disorders later in life (Yajnik \& Deshmukh 2012) providing evidence for interactions between genes and nutrients in the development of cardio-metabolic disease, including type 2 diabetes, hypertension, obesity and cardiovascular disease (CVD). The aim of this article is to provide an overview of the findings from the four studies in the gene-nutrient interactions (GeNuIne) Collaboration that used a genetic approach to explore the relationship between cardio-metabolic disease risk factors and vitamin B12 status and to investigate whether these relationships were modified by macronutrient intake (carbohydrate, fat and protein intake) (Vimaleswaran 2017).

\section{Importance of studying gene-diet interactions in different genetic groups}

It has been established that previous genetic studies looking at vitamin B12 status in healthy adults, especially large-scale ones, have been unable to capture the level of diversity which exists worldwide, as they are mainly based on individuals of European ancestry (Surendran et al. 2018). The under-representation of diverse ethnic groups hampers our full understanding of the genetic architecture of vitamin B12 concentrations (Sirugo et al. 2019). Furthermore, the limited genetic data on non-Caucasian populations in relation to genetic susceptibility to vitamin B12 deficiency can also impede our ability to translate genetic research into clinical care and will exacerbate health inequalities (Sirugo et al. 2019). Given that vitamin B12 status can also be determined by environmental factors, it is also important to explore gene-diet interactions in different ethnic groups. It is important to note that different ethnic groups respond differently to specific dietary interventions (Vimaleswaran 2017). Therefore, using estimates of genetic risk for vitamin B12 deficiency from European-based studies in non-Europeans may result in an inaccurate assessment of risk of vitamin B12 deficiency and could result in an inappropriate environmental intervention (dietary or physical activity) in under-studied populations. To address all these issues, the GeNuIne Collaboration (Vimaleswaran 2017) was initiated to investigate the effect of 
gene-lifestyle interactions on cardio-metabolic disease risk factors using population-based studies from various ethnic groups in lower-middle income countries (LMICs): Brazil (Surendran \& Vimaleswaran 2019), Sri Lanka (Surendran et al. 2019b), India (Surendran et al. 2019c) and Indonesia (Surendran et al. 2019a). Another objective of the GeNuIne Collaboration was to look at gene-lifestyle interactions on vitamin B12 concentrations (Matusheski et al. 2021). A summary of the general characteristics of the populations and the SNPs investigated are provided in Tables 1 and 2.

\section{Impact of genes and diet on homocysteine, vitamin BI2, folate and lipids in a Brazilian adolescent population}

Epidemiological studies have suggested that increased homocysteine concentrations may contribute to up to $25 \%$ of coronary events (Stanger et al. 2004) and have been shown to inversely correlate with B-complex vitamins, such as folate and vitamin B12 (Surendran \& Vimaleswaran 2019). While B-complex vitamins have a role in the reduction of homocysteine

Table I Single-nucleotide polymorphisms (SNPs) under investigation: A comparison of studies in the Brazilian, Sri Lankan, Indian and Indonesian populations

\begin{tabular}{|c|c|c|c|c|}
\hline & Brazil $(n=113)^{\mathrm{a}}$ & Sri Lanka $(n=109)$ & $\begin{array}{l}\text { India study } \\
(n=548)^{c}\end{array}$ & $\begin{array}{l}\text { Indonesia study } \\
(n=117)^{b}\end{array}$ \\
\hline $\begin{array}{l}\text { B I2-related SNPs } \\
\text { analysed }\end{array}$ & 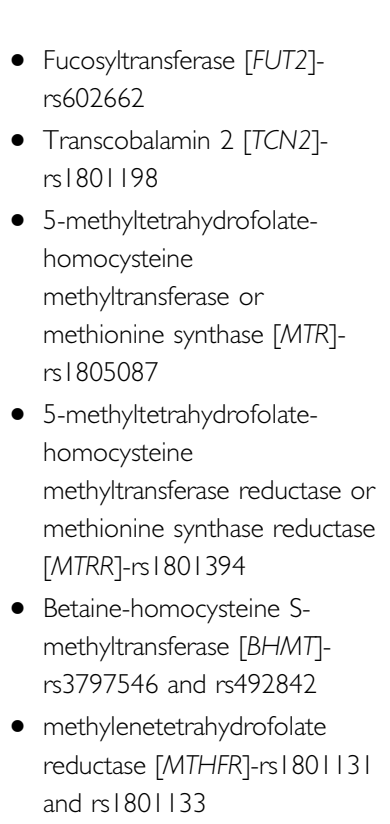 & 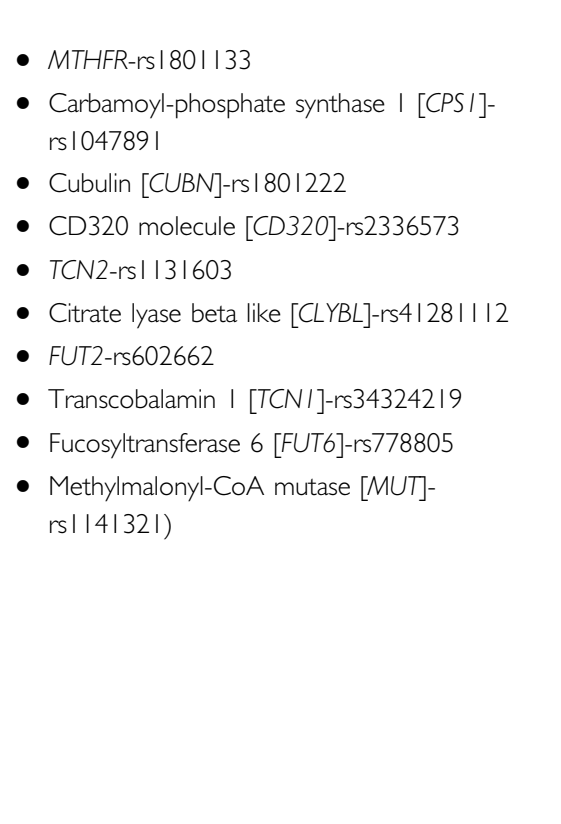 & Not applicable & 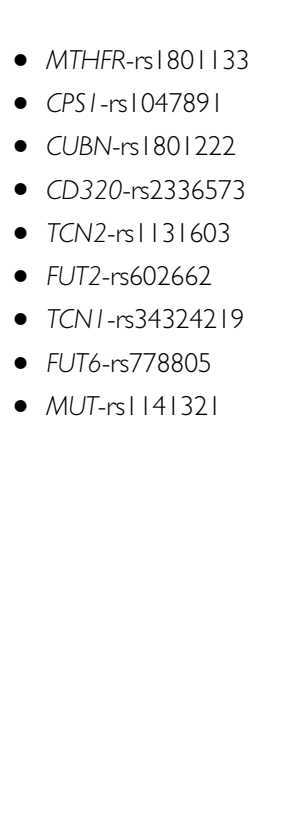 \\
\hline $\begin{array}{c}\text { Metabolic disease } \\
\text { SNPs analysed }\end{array}$ & $\begin{array}{l}\text { Catechol-o-methyl transferase } \\
\text { [COMT]-rs4680 and rs } 4633\end{array}$ & 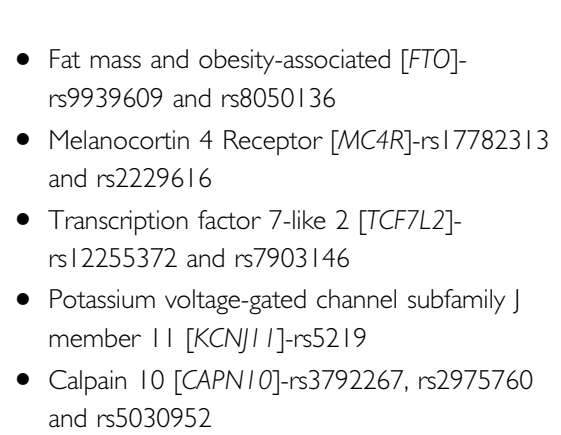 & $\begin{array}{l}\text { FTO- rs8050I36 } \\
\text { and rs2388405 }\end{array}$ & $\begin{array}{l}\text { - FTO-rs9939609 and } \\
\text { rs8050I36 } \\
\text { - } M C 4 R-r s \mid 7782313 \text { and } \\
\text { rs22296I6 } \\
\text { - } T C F 7 L 2-r s \mid 2255372 \\
\text { and rs7903।46 } \\
\text { - KCNIII-rs5219 } \\
\text { - CAPNI0-rs3792267 } \\
\text { and rs5030952 }\end{array}$ \\
\hline
\end{tabular}

${ }^{a}$ Brazilian adolescents who were overweight/obese and/or were previously diagnosed with dyslipidaemia, but not with cardiovascular disease, were included b Only women were included for the Indonesian study

${ }^{\mathrm{c}}$ Case-control study for type 2 diabetes in men and women 
Table 2 General characteristics, dietary intake and biochemical levels: A comparison of studies in the Brazilian, Sri Lankan, Indian and Indonesian populations

\begin{tabular}{|c|c|c|c|c|}
\hline & Brazil $(n=113)^{\mathrm{a}}$ & Sri Lanka study $(n=109)$ & India study $(n=548)^{c}$ & Indonesia study $(n=117)^{\mathrm{b}}$ \\
\hline Study design & Cross-sectional & Cross-sectional & Case-control & Cross-sectional \\
\hline \multicolumn{5}{|l|}{ General characteristics } \\
\hline $\mathrm{BMI}\left(\mathrm{kg} / \mathrm{m}^{2}\right)$ & $24.0 \pm 4.9$ & $24.6 \pm 4.1$ & $26.75 \pm 5.04$ & $25.1 \pm 4.2$ \\
\hline Waist-to-hip ratio & $N / A$ & $0.92 \pm 0.11$ & $0.90 \pm 0.09$ & N/A \\
\hline Fat $(\%)$ & N/A & $27.25 \pm 7.37$ & N/A & $35.70 \pm 7.00$ \\
\hline Low & 31 & 72.5 & 82.0 & 39.3 \\
\hline Medium & 69 & 19.3 & 16.2 & 49.6 \\
\hline High & & 8.3 & 1.8 & | 1.1 \\
\hline \multicolumn{5}{|l|}{ Dietary intake } \\
\hline Total energy (Kcal/day) & $2522 \pm 586$ & $2098 \pm 456$ & $2597 \pm 773$ & $1774 \pm 609$ \\
\hline Dietary carbohydrate (\%) & $47.7 \pm 20.6$ & $69.6 \pm 8.8$ & $64.3 \pm 6.3$ & $54.1 \pm 9.4$ \\
\hline 24 hours ambulatory systolic BP (mm Hg) & N/A & $120 \pm 15$ & $129 \pm 20$ & $113 \pm 9$ \\
\hline 24 hours ambulatory diastolic BP ( $\mathrm{mm} \mathrm{Hg}$ ) & $\mathrm{N} / \mathrm{A}$ & $75 \pm 16$ & $80 \pm 12$ & $77 \pm 6$ \\
\hline Triacylglycerol (mg/dl) & $94.05 \pm 54.16$ & $|44.19 \pm 86.8|$ & $146.54 \pm 1 \mid 6.88$ & $97.67 \pm 42.80$ \\
\hline $\mathrm{HDL}(\mathrm{mg} / \mathrm{dl})$ & $46.29 \pm 11.79$ & $42.56 \pm 8.24$ & $40.89 \pm 8.85$ & $58.99 \pm 10.20$ \\
\hline $\mathrm{LDL}(\mathrm{mg} / \mathrm{dl})$ & $90.28 \pm 21.00$ & $134.03 \pm 28.60$ & $1 \mid 4.37 \pm 35.56$ & $127.77 \pm 39.17$ \\
\hline VLDL (mg/dl) & $18.85 \pm 10.82$ & $28.84 \pm 17.36$ & $N / A$ & $\mathrm{~N} / \mathrm{A}$ \\
\hline Oxidised-LDL (U/I) & $6.42 \pm 13.69$ & $N / A$ & N/A & N/A \\
\hline Fasting plasma glucose (mg/dl) & N/A & $86 \pm 13$ & $116 \pm 49$ & $92 \pm 20$ \\
\hline Fasting serum insulin $(\mu \mathrm{l} \mathrm{U} / \mathrm{ml})$ & N/A & $9.9 \pm 7.2$ & $9.23 \pm 6.25$ & $32959 \pm 26327$ \\
\hline Glycated haemoglobin (\%) & N/A & $5.4 \pm 0.5$ & $6.5 \pm 1.7$ & N/A \\
\hline \multicolumn{5}{|l|}{ B-vitamin biomarker status } \\
\hline
\end{tabular}

BP, blood pressure; HDL, high density lipoprotein; LDL, low density lipoprotein; VLDL, very low density lipoprotein.

${ }^{a}$ Brazilian adolescents who were overweight/obese and/or were previously diagnosed with dyslipidaemia, but not with cardiovascular disease, were included

${ }^{b}$ Only women were included for the Indonesian study

${ }^{\mathrm{c}}$ Case-control study for type 2 diabetes in men and women

concentrations, the effect of these vitamins on cardiovascular health remains ambiguous (Ntaios et al. 2009). Some studies have suggested that high folate and vitamin B12 status are associated with a lower risk of coronary heart disease (Voutilainen et al. 2001; Ishihara et al. 2008). Common variants in genes of the one-carbon metabolism pathway have been reported to influence concentrations of homocysteine, folate, vitamin B12 and lipids (Hazra et al. 2009). The aim of this study was to examine the association of ten SNPs involved in the one-carbon metabolism pathway with vitamin B12, folic acid, homocysteine and blood lipids, and to investigate whether environmental factors (dietary factors and physical activity levels) modified the association of the SNPs in 113 Brazilian adolescents (aged 10-19 years) with cardiovascular risk (overweight or obese and/or previously diagnosed dyslipidaemia, but not with CVD).

Since several SNPs were analysed in all the studies, correction for multiple testing was applied. No genelifestyle interactions were observed on vitamin B12 concentrations. This study provided evidence for interactions between the metabolic COMT SNP rs4680 and carbohydrate intake on ox-LDL concentrations and the B12-associated FUT2 SNP rs602662 and protein intake on homocysteine concentrations. This is the first study to provide novel gene-diet interactions at the COMT and FUT2 gene loci, on ox-LDL and 
homocysteine concentrations; hence, we have no other studies to compare our findings with. Given that oxLDL and homocysteine are well-known independent risk factors for cardiovascular disease (Shenoy et al. 2014; Papageorgiou \& Tousoulis 2016), these findings may have significant public health implications.

In addition to the genetic component of metabolic traits and markers of one-carbon metabolites, physical inactivity could be an important contributor that could interact with an individual's genetic predisposition. Our results showed that the MTHFR SNP rs1801131 showed a significant interaction with physical activity on folic acid concentrations. Folate plays a critical role in the methylation pathway and is involved in the methylation of DNA, creatine and acetylcholine, all of which are important for physical activity. It is important to note that physical activity levels may interact with folate metabolism by increasing intestinal folate absorption or stimulating the methionine synthase enzyme due to an increased metabolic demand and the associated possible increase in turnover of methylated molecules required for exercise (Kim et al. 2016). It is important that in the future, replications of this finding are made, preferably in a larger independent cohort with adequate statistical power utilising more direct measures of physical activity.

\section{Impact of genes and diet on vitamin B I 2 concentrations and cardio-metabolic diseases in an Asian Sri Lankan population (GOOD study)}

South Asians exhibit a unique phenotype collectively known as the 'South Asian Phenotype' which consists of higher levels of total and visceral fat, higher waist circumference and an increased susceptibility to type 2 diabetes (Mohan \& Deepa 2006; Surendran et al. $2019 b$ ). Currently, over $34.4 \%$ of the Sri Lankan adult population are diagnosed as being overweight or obese (Katulanda et al. 2010). Although there is a strong genetic component to developing the 'South Asian Phenotype', consuming an unhealthy diet and leading a sedentary lifestyle can further contribute to this phenotype (Anjana et al. 2014; Vimaleswaran et al. 2016). Several studies have suggested that obesity is related to many micronutrient deficiencies including vitamin B12 (Astrup \& Bügel 2010; Damms-Machado et al. 2012; Chakraborty et al. 2018). This is the first study to investigate the relationship between metabolic traits and vitamin B12 status using a gene-based approach in 109 Sinhalese adults [Genetics of Obesity and Diabetes (GOOD) study; 61 men and 48 women, aged 25-50 years], and to investigate whether these relationships were modified by lifestyle factors (dietary factors/physical activity levels).

The unweighted genetic risk score (GRS) was calculated for each participant based on the sum of risk allele counts across each SNP which predicted a lower vitamin B12 status (B12-GRS). A second, unweighted GRS was created using allele markers previously reported to be associated with metabolic disease traits (metabolic-GRS). A numerical value of 0,1 or 2 was assigned to each SNP, which indicates the number of risk alleles on that SNP. The scores were then calculated by adding the number of risk alleles across each SNP. The risk allele score was then divided by the median score into those carrying $\leq 9$ risk alleles versus those with $\geq 10$ risk alleles for the B12 GRS and $\leq 8$ risk alleles versus those with $\geq 9$ risk alleles for the metabolic-GRS.

The findings from this study demonstrated that a genetically lowered vitamin B12 concentration may be associated with central obesity (waist circumference) in the presence of a dietary influence (protein energy intake \%). Additionally, the metabolic-GRS interacted with carbohydrate energy intake $(\%)$ to influence waist-to-hip ratio levels, where individuals carrying more than 9 risk alleles had a higher waist-to-hip ratio among those in the highest tertile of carbohydrate energy percentage compared to those in the lowest tertile (mean $\pm \mathrm{SD}=78.00 \pm 7.90 \%$; Fig. 1$)$.

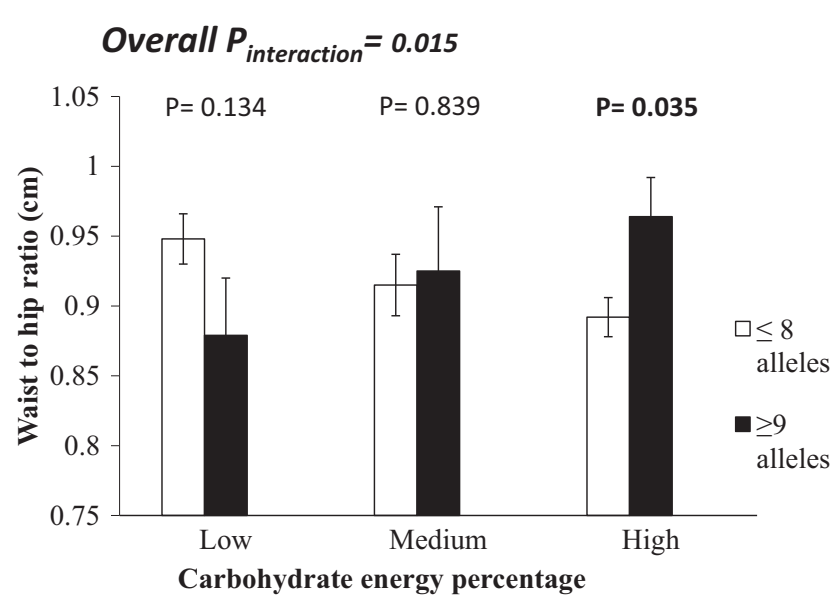

Figure I Interaction between the metabolic-genetic risk score and carbohydrate energy intake (\%) on waist-to-hip ratio $\left(P_{\text {interaction }}=0.015\right)$. Among those who consumed a high carbohydrate diet, individuals who carried nine or more risk alleles had significantly higher waist-to-hip ratios compared to individuals carrying eight or less risk alleles $(P=0.035)$ 
Further studies are warranted to investigate the mechanisms underlying the effect of these GRSs on metabolic traits. Given that the daily consumption of protein is low and carbohydrate intake is high in Sri Lankan adults (Jayawardena et al. 2014), these findings, if confirmed in randomised control trials, may have significant public health implications in terms of revising dietary guidelines for this population, which could prevent central obesity and its related CVD-related outcomes.

\section{Impact of genes and diet on vitamin B I 2 concentrations and metabolic diseases in an Asian Indian population (CURES study)}

Although vitamin B12 deficiency has been linked to obesity (Pinhas-Hamiel et al. 2006; MacFarlane et al. 2011; Baltaci et al. 2013) and diabetes (Yajnik et al. 2008; Krishnaveni et al. 2009; Knight et al. 2015), no study to date has tested the genetic link between metabolic traits and vitamin B12 status in an Asian Indian population (Surendran et al. 2019c). Hence, this study examined whether dietary intake and physical activity levels modified associations between a GRS using two previously studied FTO SNPs (rs8050136 and rs2388405) and vitamin B12 concentrations and metabolic disease-related outcomes in 548 Asian Indians (220 normal glucose-tolerant individuals, 152 individuals with prediabetes and 176 individuals with type 2 diabetes). In this study, participants were randomly recruited from the Chennai Urban Rural Epidemiology Study (CURES), a cross-sectional case-control epidemiological study conducted on a representative population of Chennai in Southern India (Deepa et al. 2003).

To date, the FTO (fat mass and obesity associated) gene has been the strongest obesity risk loci in several populations (Vimaleswaran \& Loos 2010). Thus, the FTO gene was considered as a suitable candidate to establish the genetic link between obesity-related traits and vitamin B12 concentrations and to determine whether this relationship was modified by lifestyle factors. We found no significant interactions between the FTO-GRS and lifestyle factors (diet and physical activity levels) in this study. However, a novel finding of this study indicated the potential association between the FTO-GRS and vitamin B12 concentrations, after adjustment for potential confounders. Carriers of more than one risk allele for the FTO-GRS had lower vitamin B12 concentrations (mean $\pm \mathrm{SD}: 355 \pm 189 \mathrm{pg} / \mathrm{ml}$ ), compared to individuals carrying zero risk alleles (mean \pm SD: $\quad 410 \pm 202 \mathrm{pg} / \mathrm{ml}$ ). The mechanism explaining the differences in vitamin B12 concentrations in the FTO-GRS could potentially be due to the FTO genotypes modulating gut microbiota and inducing metabolic inflammation, consequently impairing B12 absorption (Caesar et al. 2015; Chakraborty et al. 2018).

Given that low vitamin B12 concentrations in Asian Indians are common (Yajnik et al. 2006; Sivaprasad et al. 2016) and that 28-44\% of Asians carry at least one copy of the FTO risk allele ( $\mathrm{Li}$ et al. 2012), this study highlights the importance of considering obesity as a risk factor for vitamin B12 deficiency. This has implications for the possible targeting of relevant obesity prevention strategies, by focusing on increasing vitamin B12 intakes in obese or overweight individuals. Following such advice could substantially reduce vitamin B12 deficiency among Asian Indians.

\section{Impact of genes and diet on vitamin B I 2 concentrations and metabolic diseases in an Indonesian women population (Minangkabau community: MINANG study)}

Optimal vitamin B12 status is essential for women to maintain adequate maternal health and to avoid fetal developmental complications (Krishnaveni et al. 2009; Dror \& Allen 2012; Surendran et al. 2019a). Additionally, low vitamin B12 concentrations have shown negative correlations with BMI in healthy women (Baltaci et al. 2017). Currently, the prevalence of low vitamin B12 status in healthy Indonesian women is unknown and studies on the relationship between low vitamin B12 status and obesity-related traits have yielded conflicting results (Wiebe et al. 2018). The Minangkabau population from Indonesia is of particular interest, as it is the largest matrilineal society in the world. Women in this society have relatively greater authority within the family compared to men and have a major role in dictating food choices and maintaining the welfare of the family (Stark 2013). In this population, GRSs based on nine B12-related SNPs and nine metabolic disease-related SNPs were constructed.

In the Indonesian study, a novel interaction between the B12-GRS and dietary fibre intake $(\mathrm{g})$ on glycated haemoglobin was observed $\left(P_{\text {interaction }}=0.042\right.$; Fig. 2). Individuals who consumed a low-fibre diet $(4.90 \pm 1.00 \mathrm{~g} / \mathrm{day})$ and carried $\geq 9$ risk alleles for vitamin B12 deficiency had significantly higher HbA1C concentrations compared to those carrying $\leq 8$ risk alleles. 


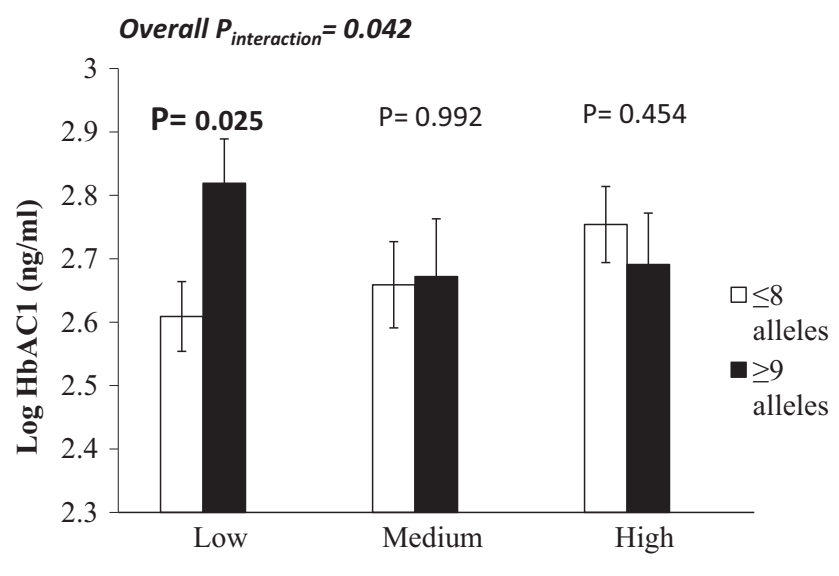

Tertiles of dietary fibre intake

Figure 2 Interaction between the BI2-genetic risk score and dietary fibre intake (g) on log $\mathrm{HbACl}\left(\mathrm{ng} / \mathrm{ml} ; P_{\text {interaction }}=0.042\right)$. Among those who consumed a low-fibre diet, individuals who carried 9 or more risk alleles had significantly higher concentrations of $\log \mathrm{HbACl}$ compared to individuals carrying eight or less risk alleles $(P=0.025)$

Furthermore, interactions were also seen between the B12-GRS and protein (energy \%) on log transformed body fat percentage.

Previous studies have shown that dietary fibre consumption is low in the Indonesian population, and thus, this finding, if confirmed in randomised controlled trials, could be the basis of a consumer education campaign centred around encouraging fibre intake to reduce $\mathrm{HbA} 1 \mathrm{C}$ concentrations with those with genetic risk, which could improve glycaemic control in this population.

\section{Ethnic-specific differences}

The observations described in these studies confirm previous findings on population-based differences in vitamin B12 concentrations (McLean et al. 2008). The two South Asian populations demonstrated lower vitamin B12 concentrations than the Indonesian women population [Indian $(417 \pm 255 \mathrm{pg} / \mathrm{ml})$ and Sri Lankan populations $(516 \pm 180 \mathrm{pg} / \mathrm{ml})$ vs. Indonesian women $(591 \pm 579 \mathrm{pg} / \mathrm{ml})]$. Despite the Brazilian adolescent population exhibiting signs of cardiovascular risk, intermediate vitamin B12 concentrations were observed in this population $(520 \pm 232 \mathrm{pg} / \mathrm{ml})$. It is difficult to generalise these findings meaningfully, as some of the populations included cohorts at risk of disease and furthermore the sample size of the population was limited (Table 2).

Several genetic loci have supported the presence of ethnic differences for metabolic traits and vitamin B12 status within the populations studied in this review. The FTO rs8050136 genotype is an example of genetic heterogeneity according to race. The minor allele 'A' of the SNP rs8050136 was present in 13\% of Indian participants versus $23 \%$ of Indonesian participants and although India and Sri Lanka are geographically close, it was even more frequent in the Sinhalese population $(34 \%)$.

The FUT2 SNP rs602662 is one of the most commonly studied variants related to vitamin B12 status. The rs602662 SNP is an example of a B12 SNP that has demonstrated ethnic specificity, for example within the Indonesian population the minor allele frequency was extremely low $(0.03 \%)$ in comparison to the Sri Lankan $(31 \%)$ and Brazilian populations $(41 \%)$. Although no genotyping errors were identified, the SNP rs602662 did not reach Hardy-Weinberg equilibrium (HWE), when using the chi-square test, within the Indonesian and Sri Lankan populations. It is possible that HWE was not reached in these populations due to the small sample size and the possibility of interbreeding (especially as consanguineous marriages are common in these populations).

\section{How these findings could be translated into effective personalised and public health strategies}

Obesity and perhaps vitamin B12 deficiency are important modifiable risk factors for a variety of chronic diseases. Obesity and vitamin B12 status are known to be associated, but the direction of the association and whether it is a causal relationship has been uncertain. Many genetic variants have been identified for vitamin B12 deficiency and metabolic diseases through candidate gene and genome-wide approaches (Bradfield et al. 2012; Willer et al. 2013; Surendran et al. 2018). However, it is possible that these genetic variants may not cause diseases without exposure to certain dietary factors (Rhee et al. 2012). Although this study has shown that low vitamin B12 concentrations are associated with metabolic diseases through a dietary interaction, the molecular and pathophysiological mechanisms remain unknown. It is important that mechanistic studies are carried out to determine how genetically low vitamin B12 concentrations interact with dietary factors to influence adipose tissue metabolism or how epigenetic mechanisms contribute to the development of metabolic diseases. Current literature also suggests that the genetic profile of an individual may shape the microbiome of the host, and an altered gut flora has been associated with vitamin B12 deficiency (Hall et al. 


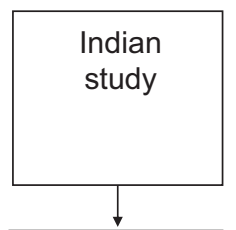

The FTO GRS

was

associated

with B12

concentrations

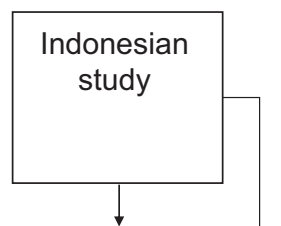

The B12-GRS

interacted

with fibre

intake on

HbA1C levels

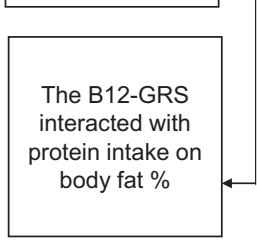

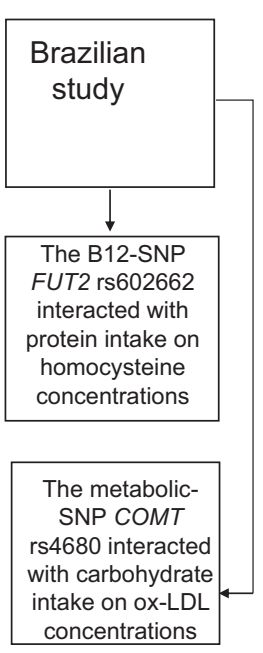

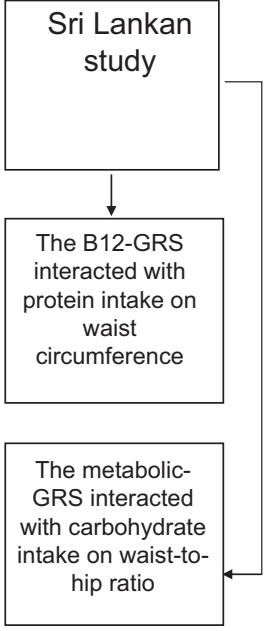

Overall, the genetic risk of vitamin B12 deficiency may be associated with metabolic traits through a dietary influence (protein and fibre intake)

Figure 3 The main study findings of the GeNulne Collaboration

2017; Surendran et al. 2018). This possibility requires investigation, given the known link between an altered distribution in gut microbiota and obesity. Hence, the combined application of nutrigenetics, nutrigenomics [dietary effects on gene expression (Ordovas et al. 2018)] and metabolomics (dietary effects on metabolite changes) is needed to clarify the gene-diet interactions identified in this project.

However, before the findings from these studies can be translated into personalised advice and/or public health strategies, they require confirmation from larger observational studies which include individuals from different ethnic groups and randomised controlled trials. It is difficult to truly isolate the macronutrient accountable for any nutrigenetic effects in observational studies, especially for fat and carbohydrates, as one macronutrient usually compensates the other (Sarzynski \& Bouchard 2013). Therefore, dietary intervention studies are preferable as they can manipulate dietary intake more specifically. Dietary intervention studies also have the potential to avoid the measurement bias found within food frequency questionnaires and can demonstrate causal relationships between SNPs, diet and metabolic traits. Future studies should also measure intakes of specific dietary micronutrients to enable more detailed investigations of gene-diet interactions to be carried out. More studies that robustly measure both body composition (e.g. dual-energy X-ray absorptiometry, magnetic resonance imaging and/or computed tomography scans) and body size, and which measure vitamin B12 status using markers such as homocysteine, MMA and holotranscobalamin to investigate the effects of adiposity on vitamin B12 status across all body sizes are also needed (Wiebe et al. 2018).

\section{Conclusion}

In conclusion, the GeNuIne Collaboration identified novel interactions between vitamin B12-related genes and dietary factors (in particular protein intake and fibre intake) on cardio-metabolic traits within Brazilian, Sri Lankan and Indonesian populations. In addition, within an Indian population, individuals who were genetically predisposed to obesity had lower vitamin B12 concentrations, without influence from dietary factors (Fig. 3). Further understanding of the role of these gene-diet interactions at the molecular level is needed before diets can be tailored according to each ethnic subgroup.

\section{Acknowledgements}

Dr Karani S Vimaleswaran acknowledges support from the British Nutrition Foundation through the Drummond Pump Priming Award and thanks the support from the authors of the GeNuIne Collaboration (Vimaleswaran 2020). 


\section{Conflict of interest}

The authors have no conflict of interest.

\section{References}

Adaikalakoteswari A, Jayashri R, Sukumar N et al. (2014) Vitamin B12 deficiency is associated with adverse lipid profile in Europeans and Indians with type 2 diabetes. Cardiovascular Diabetology 13: 1-7.

Allen, LH, 2008. Causes of vitamin B12 and folate deficiency. Food and Nutrition Bulletin 29(2_suppl1), pp. S20-S34.

Allin KH, Friedrich N, Pietzner M et al. (2017) Genetic determinants of serum vitamin B12 and their relation to body mass index. European Journal of Epidemiology 32: 125-34.

Anjana RM, Pradeepa R, Das AK et al. (2014) Physical activity and inactivity patterns in India-results from the ICMR-INDIAB study (Phase-1)[ICMR-INDIAB-5]. International Journal of Behavioral Nutrition and Physical Activity 11: 1-11.

Astrup A \& Bügel S (2010) Micronutrient deficiency in the aetiology of obesity. International Journal of Obesity 34: 947-8.

Baltaci D, Deler MH, Turker Y et al. (2017) Evaluation of serum vitamin B12 level and related nutritional status among apparently healthy obese female individuals. Nigerian Journal of Clinical Practice 20: 99-105.

Baltaci D, Kutlucan A, Turker Y et al. (2013) Association of vitamin B12 with obesity, overweight, insulin resistance and metabolic syndrome, and body fat composition; primary care-based study. Medicinski Glasnik 10: 203-10.

Bradfield JP, Taal HR, Timpson NJ et al. (2012) A genome-wide association meta-analysis identifies new childhood obesity loci. Nature Genetics 44: 526.

Caesar R, Tremaroli V, Kovatcheva-Datchary P et al. (2015) Crosstalk between gut microbiota and dietary lipids aggravates WAT inflammation through TLR signaling. Cell Metabolism 22: 658-68.

Chakraborty S, Chopra M, Mani K et al. (2018) Prevalence of vitamin B12 deficiency in healthy Indian school-going adolescents from rural and urban localities and its relationship with various anthropometric indices: a cross-sectional study. Journal of Human Nutrition and Dietetics 31: 513-22.

Damms-Machado A, Weser G \& Bischoff SC (2012) Micronutrient deficiency in obese subjects undergoing low calorie diet. Nutrition Journal 11: 1-10.

Deepa M, Pradeepa R, Rema M et al. (2003) The Chennai Urban Rural Epidemiology Study (CURES)-study design and methodology (urban component)(CURES-I). The Journal of the Association of Physicians of India 51: 863-70.

Dror DK \& Allen LH (2012) Interventions with vitamins B6, B12 and $\mathrm{C}$ in pregnancy. Paediatric and Perinatal Epidemiology 26: 55-74.

Green R, Allen LH, Bjørke-Monsen AL et al. (2017) Vitamin B 12 deficiency. Nature Reviews Disease Primers 3: 1-20.

Guarnizo-Poma M, Urrunaga-Pastor D, Montero-Suyo C et al. (2018) Association between serum vitamin B12 levels and metabolic syndrome in a euthyroid population. Diabetes \& Metabolic Syndrome: Clinical Research \& Reviews 12: 943-8.

Hall AB, Tolonen AC \& Xavier RJ (2017) Human genetic variation and the gut microbiome in disease. Nature Reviews Genetics 18: 690-9.
Hazra A, Kraft P, Lazarus R et al. (2009) Genome-wide significant predictors of metabolites in the one-carbon metabolism pathway. Human Molecular Genetics 18: 4677-87.

Husemoen LLN, Skaaby T, Thuesen BH et al. (2016) Mendelian randomisation study of the associations of vitamin B12 and folate genetic risk scores with blood pressure and fasting serum lipid levels in three Danish population-based studies. European Journal of Clinical Nutrition 70: 613-9.

Institute of Medicine (US) Standing Committee on the Scientific Evaluation of Dietary Reference Intakes and its Panel on Folate, Other B Vitamins, and Choline (2000) Dietary Reference Intakes for Thiamin, Riboflavin, Niacin, Vitamin B6, Folate, Vitamin B12, Pantothenic Acid, Biotin, and Choline. National Academies Press: Washington.

Ishihara J, Iso $\mathrm{H}$, Inoue $\mathrm{M}$ et al. (2008) Intake of folate, vitamin B6 and vitamin B12 and the risk of CHD: the Japan Public Health Center-Based Prospective Study Cohort I. Journal of the American College of Nutrition 27: 127-36.

Jayashri R, Venkatesan U, Rohan M et al. (2018) Prevalence of vitamin B 12 deficiency in South Indians with different grades of glucose tolerance. Acta Diabetologica 55: 1283-93.

Jayawardena R, Thennakoon S, Byrne N et al. (2014) Energy and nutrient intakes among Sri Lankan adults. International Archives of Medicine 7: 1-11.

Katulanda P, Jayawardena MAR, Sheriff MHR et al. (2010) Prevalence of overweight and obesity in Sri Lankan adults. Obesity Reviews 11: 751-6.

Kim YN, Hwang JH \& Cho YO (2016) The effects of exercise training and acute exercise duration on plasma folate and vitamin B12. Nutrition Research and Practice 10: 161.

Knight BA, Shields BM, Brook A et al. (2015) Lower circulating B12 is associated with higher obesity and insulin resistance during pregnancy in a non-diabetic white British population. PLoS One 10: e0135268.

Koury MJ \& Ponka P (2004) New insights into erythropoiesis: the roles of folate, vitamin B12, and iron. Annual Review of Nutrition 24: 105-31.

Krishnaveni GV, Hill JC, Veena SR et al. (2009) Low plasma vitamin B 12 in pregnancy is associated with gestational 'diabesity'and later diabetes. Diabetologia 52: 2350-8.

Kumar KA, Lalitha A, Pavithra D et al. (2013) Maternal dietary folate and/or vitamin B12 restrictions alter body composition (adiposity) and lipid metabolism in Wistar rat offspring. The Journal of Nutritional Biochemistry 24: 25-31.

Li H, Kilpeläinen TO, Liu C et al. (2012) Association of genetic variation in FTO with risk of obesity and type 2 diabetes with data from 96,551 East and South Asians. Diabetologia 55: 98195.

MacFarlane AJ, Greene-Finestone LS \& Shi Y (2011) Vitamin B-12 and homocysteine status in a folate-replete population: results from the Canadian Health Measures Survey. The American Journal of Clinical Nutrition 94: 1079-87.

Mahalle N, Kulkarni MV, Garg MK et al. (2013) Vitamin B12 deficiency and hyperhomocysteinemia as correlates of cardiovascular risk factors in Indian subjects with coronary artery disease. Journal of Cardiology 61: 289-94.

Matusheski N, Caffrey A, Christensen L et al. (2021) Diets, nutrients, genes and the microbiome: recent advances in personalised 
nutrition. British Journal of Nutrition 125: 1-9. https://oi.org/10. 1017/S0007114521000374

Mclean E, De Benoist B \& Allen LH (2008) Review of the magnitude of folate and vitamin B12 deficiencies worldwide. Food and Nutrition Bulletin 29: S38-51.

Moen GH, Qvigstad E, Birkeland KI et al. (2018) Are serum concentrations of vitamin B-12 causally related to cardiometabolic risk factors and disease? A Mendelian randomization study. The American Journal of Clinical Nutrition 108: 398-404.

Mohan V \& Deepa R (2006) Adipocytokines and the expanding'Asian Indian Phenotype'. The Journal of the Association of Physicians of India 54: 685-6.

Narang M, Singh M \& Dange S (2016) Serum homocysteine, vitamin B12 and folic acid levels in patients with metabolic syndrome. The Journal of the Association of Physicians of India 64: 22-6.

Ntaios G, Savopoulos C, Grekas D et al. (2009) The controversial role of B-vitamins in cardiovascular risk: an update. Archives of Cardiovascular Diseases 102: 847-54.

O'Leary F \& Samman S (2010) Vitamin B12 in health and disease. Nutrients 2: 299-316.

Ordovas JM, Ferguson LR, Tai ES et al. (2018) Personalised nutrition and health. British Medical Journal 361: 1-6.

Papageorgiou N \& Tousoulis D (2016) Oxidized-LDL immunization for the treatment of atherosclerosis: how far are we? International Journal of Cardiology 222: 93-4.

Pinhas-Hamiel O, Doron-Panush N, Reichman B et al. (2006) Obese children and adolescents: a risk group for low vitamin B12 concentration. Archives of Pediatrics \& Adolescent Medicine 160: 933-6.

Rhee KE, Phelan S \& McCaffery J (2012) Early determinants of obesity: genetic, epigenetic, and in utero influences. International Journal of Pediatrics 2012: 1-9.

Sarzynski MA \& Bouchard C (2013) The challenging chase for nutrigenetic predictors of metabolic responses to dietary interventions. Diabetes Care 36: 3379-81.

Selhub J (2002) Folate, vitamin B12 and vitamin B6 and one carbon metabolism. The Journal of Nutrition, Health \& Aging 6: 39-42.

Shenoy V, Mehendale V, Prabhu K et al. (2014) Correlation of serum homocysteine levels with the severity of coronary artery disease. Indian Journal of Clinical Biochemistry 29: 339-44.

Sirugo G, Williams SM \& Tishkoff SA (2019) The missing diversity in human genetic studies. Cell 177: 26-31.

Sivaprasad M, Shalini T, Balakrishna N et al. (2016) Status of vitamin B12 and folate among the urban adult population in South India. Annals of Nutrition and Metabolism 68: 94-102.

Smith AD, Warren MJ \& Refsum H (2018) Vitamin B12. Advances in Food and Nutrition Research 83: 215-79.

Sobczyńska-Malefora A, Gorska R, Pelisser M et al. (2014) An audit of holotranscobalamin ("Active" B12) and methylmalonic acid assays for the assessment of vitamin B12 status: application in a mixed patient population. Clinical biochemistry 47: 82-6.

Stabler SP \& Allen RH (2004) Vitamin B12 deficiency as a worldwide problem. Annual Review of Nutrition 24: 299-326.

Stanger O, Herrmann W, Pietrzik K et al. (2004) Clinical use and rational management of homocysteine, folic acid, and $\mathrm{B}$ vitamins in cardiovascular and thrombotic diseases. Zeitschrift für Kardiologie 93: 439-53.
Stark A (2013) The matrilineal system of the Minangkabau and its persistence throughout history: a structural perspective. Southeast Asia: A Multidisciplinary Journal 13: 1-13.

Sun Y, Sun M, Liu B et al. (2019) Inverse association between serum vitamin B12 concentration and obesity among adults in the United States. Frontiers in Endocrinology 10: 414.

Surendran S, Adaikalakoteswari A, Saravanan P et al. (2018) An update on vitamin B12-related gene polymorphisms and B12 status. Genes \& Nutrition 13: 1-35.

Surendran S, Aji AS, Ariyasra U et al. (2019a) A nutrigenetic approach for investigating the relationship between vitamin B12 status and metabolic traits in Indonesian women. Journal of Diabetes \& Metabolic Disorders 18: 389-99.

Surendran S, Alsulami S, Lankeshwara R et al. (2019b) A genetic approach to examine the relationship between vitamin $B$ 12 status and metabolic traits in a South Asian population. International Journal of Diabetes in Developing Countries 40: 21-31.

Surendran S, Jayashri R, Drysdale L et al. (2019c) Evidence for the association between FTO gene variants and vitamin B12 concentrations in an Asian Indian population. Genes \& Nutrition 14: $1-9$.

Surendran S, Morais, CC, Abdalla, DSP, Shatwan, IA, Lovegrove, JA, Cominetti, C, Vimaleswaran, VK \& Horst, MA. (2019) The influence of one-carbon metabolism gene polymorphisms and gene-environment interactions on homocysteine, Vitamin B12, folate and lipids in a Brazilian adolescent population. Journal of Diabetology 10: 110-22.

Thomas-Valdés S, Tostes MDGV, Anunciação PC et al. (2017) Association between vitamin deficiency and metabolic disorders related to obesity. Critical Reviews in Food Science and Nutrition 57: 3332-43.

Vimaleswaran KS (2017) Gene-nutrient interactions on metabolic diseases: findings from the GeNuIne Collaboration. Nutrition Bulletin 42: 80-6.

Vimaleswaran KS (2020) A nutrigenetics approach to study the impact of genetic and lifestyle factors on cardiometabolic traits in various ethnic groups: findings from the GeNuIne Collaboration. The Proceedings of the Nutrition Society 79: 194-204.

Vimaleswaran KS, Bodhini D, Lakshmipriya $\mathrm{N}$ et al. (2016) Interaction between FTO gene variants and lifestyle factors on metabolic traits in an Asian Indian population. Nutrition \& Metabolism 13: $1-10$.

Vimaleswaran KS \& Loos RJ (2010) Progress in the genetics of common obesity and type 2 diabetes. Expert Reviews in Molecular Medicine 12: e7.

Voutilainen S, Rissanen TH, Virtanen J et al. (2001) Low dietary folate intake is associated with an excess incidence of acute coronary events: The Kuopio Ischemic Heart Disease Risk Factor Study. Circulation 103: 2674-80.

Wall C (1998) Food and Nutrition Guidelines for Healthy Adolescents. Ministry of Health: Wellington, New Zealand.

Wiebe N, Field CJ \& Tonelli M (2018) A systematic review of the vitamin B12, folate and homocysteine triad across body mass index. Obesity Reviews. 19: 1608-18

Willer CJ, Schmidt EM, Sengupta S et al. (2013) Discovery and refinement of loci associated with lipid levels. Nature Genetics 45: 1274 . 
Yajnik CS \& Deshmukh US (2012) Fetal programming: maternal nutrition and role of one-carbon metabolism. Reviews in Endocrine and Metabolic Disorders 13: 121-7.

Yajnik C, Deshpande SS, Lubree HG et al. (2006) Vitamin B12 deficiency and hyperhomocysteinemia in rural and urban Indians. The Journal of the Association of Physicians of India 54: 82.
Yajnik CS, Deshpande SS, Jackson AA et al. (2008) Vitamin B 12 and folate concentrations during pregnancy and insulin resistance in the offspring: the Pune Maternal Nutrition Study. Diabetologia 51: $29-38$. 